\title{
Erratum to: Quantification of Dermatophyte Viability for Evaluation of Antifungal Effect by Quantitative PCR
}

\section{Tomoyuki Iwanaga $\cdot$ Kazushi Anzawa \\ Takashi Mochizuki}

Published online: 9 December 2014

(C) Springer Science+Business Media Dordrecht 2014

Erratum to: Mycopathologia (2014) 177:241-249 DOI 10.1007/s11046-014-9745-5

Because of a mistake during the editorial process, the given names and family names of all three authors are shown in the wrong order. They should be Tomoyuki Iwanaga, Kazushi Anzawa and Takashi Mochizuki.

The online version of the original article can be found under doi:10.1007/s11046-014-9745-5.

T. Iwanaga $(\bowtie)$

R\&D Laboratories, Pola Pharma Inc., 560 Kashio-cho,

Totuka-ku, Yokohama 244-0812, Japan

e-mail: t-iwanaga@pola-pharma.co.jp;

t-iwanag@kanazawa-med.ac.jp

T. Iwanaga $\cdot$ K. Anzawa $\cdot$ T. Mochizuki

Department of Dermatology, Kanazawa Medical

University, Uchinada, Ishikawa, Japan 